\title{
Sobretensiones en Arrancadores con Autotransformador Causadas por la Desconexión del Interruptor Principal en el Arranque de los Motores
}

\author{
Arturo García, Miguel A. Zapata, Javier A. Estrada y Job García \\ Instituto Nacional de Electricidad y Energías Limpias, Reforma 113, Col Palmira, Cuernavaca, Morelos, \\ CP 62490, México. (e-mail; tevillo@ineel.mx; mzapata@ineel.mx; jaeg@ineel.mx; jgarcia@ineel.mx)
}

Recibido Jul. 21, 2017; Aceptado Oct. 5, 2017; Versión final Nov. 29, 2017, Publicado Jun. 2018

\begin{abstract}
Resumen
Se discuten tres fallas, que se presentaron en el lado línea, de tres arrancadores con autotransformador de $13.8 \mathrm{kV}$. Las fallas fueron ocasionadas por sobretensiones, generadas por el fenómeno de re-encendidos múltiples y escalación de tensiones, el cual, a su vez, fue ocasionado por la apertura del interruptor principal, durante el proceso de arranque de los motores. Este mecanismo de falla es diferente a los que han sido reportados en la literatura especializada, ocasionados por la apertura del interruptor del neutro o por el cierre del interruptor principal, durante el arranque de los motores. Para proteger a los arrancadores contra las sobretensiones en el lado línea, es necesario instalar, en esta zona, una combinación de apartarrayos y supresores de transitorios RC. Los apartarrayos cortan la magnitud de la sobretensión y los supresores modifican su frecuencia. Esto minimiza las posibilidades de que se generen re-encendidos múltiples y escalación de tensiones.
\end{abstract}

Palabras clave: método Korndörfer; arrancador con autotransformador; re-encendidos múltiples; escalación de tensiones; fallas en arranque

\section{Overvoltages in Autotransformer Starters Caused by the Opening of the Main Circuit Breaker in the Motors Starting}

\begin{abstract}
Three faults, localized in the line side of three $13.8 \mathrm{kV}$ autotransformer starters, are discussed. The causes of the failures were overvoltages, generated by the multiple re-ignition and voltage escalation phenomenon, generated by the opening of the main switch during the motors starting. This fault mechanism is different from those reported in the specialized literature, due to the opening of the neutral switch and the closing of the main switch, during the motors starting. To protect the starters against overvoltages in the line side of the transformers, a combination of surge arresters and RC transient suppressors, is required in this zone. Arresters limit the magnitude of the overvoltages and RC suppressors modify their frequency. This minimizes the chances of multiple re-ignitions and voltage escalation.
\end{abstract}

Key words: Korndörfer method; autotransformer starters; multiple re-ignitions; voltage escalation; failures in the motor starting 


\section{INTRODUCCIÓN}

El método Korndörfer o sistema de arranque con autotransformador, a tensión reducida (Sistema ATR), se utiliza para disminuir la corriente de arranque de motores de gran capacidad (Hubbi y Halak, 1994; Lawrence y Thomas, 2007). Los Sistemas ATR han sido ampliamente utilizados en baja tensión. Sin embargo, cuando la aplicación de estos sistemas se extendió a niveles de media tensión (de 2.4 a $15 \mathrm{kV}$ ), empezaron a reportarse diversas fallas, en la década de los setentas (Lawrence y Arthur, 2004). Durante el proceso de arranque de los motores, se detectaron sobretensiones, aparentemente inexplicables en los Sistemas ATR, en el lado neutro de los autotransformadores. Estas sobretensiones, generaban corto circuitos en el autotransformador o arcos eléctricos entre las zonas energizadas y el gabinete, conectado a tierra, tal como se muestra en la Fig. 1.

Al desconocer la causa-raíz de las fallas, los Sistemas ATR comenzaron a ser retirados de servicio. Las publicaciones técnicas de los Sistemas ATR también disminuyeron. Solamente los usuarios con una gran cantidad de Sistemas ATR, cuyos motores no podían arrancar a tensión plena, siguieron utilizando estos equipos, buscando la manera de incrementar su confiabilidad.

A finales del siglo $X X$ y a principios del siglo XXI, se reportó que las maniobras de los interruptores de vacío, generaban sobre tensiones aleatorias considerables (Telander et al, 1987; Chaly y Chalaya, 1996; IEC 60470, 2000). Esto ayudó a determinar la causa-raíz de las fallas en los Sistemas ATR. Se encontró que, al abrir el interruptor del neutro, durante el proceso de arranque, se generan sobre tensiones aleatorias, ocasionadas por re-igniciones múltiples y escalación de tensiones, las cuales, a su vez, generan fallas dieléctricas (Lawrence y Arthur, 2004). También se determinó que los apartarrayos y los supresores de transitorios tipo C (capacitor conectado entre línea y tierra) no protegen contra este tipo de sobre tensiones (Telander et al, 1987; Ouahdi, 1991).

Las fallas en los Sistemas ATR, se lograron minimizar, instalando en el neutro, una combinación de apartarrayos y supresores de transitorios tipo RC (un capacitor y una resistencia conectados en serie, entre línea y tierra) (Lawrence y Arthur, 2004; Vollet y De Metz Nobat, 2007). Los apartarrayos reducen la sobre tensión y los supresores de transitorios RC modifican su frecuencia. Esto minimiza la posibilidad de reencendidos múltiples y la escalación de tensiones (Emam, et al, 2008). Los apartarrayos y los supresores de transitorios RC, quedaron instalados dentro del gabinete de los Sistemas ATR. Esta medida correctiva se generalizó en el panorama mundial, en el período de 2004 a 2007, demostrando tácitamente su efectividad y su rentabilidad. Inclusive, algunos usuarios instalaron apartarrayos y supresores de transitorios $\mathrm{RC}$ tanto en el lado neutro de los autotransformadores, como en las terminales del motor.

Con la medida correctiva, los Sistemas ATR presentaron 8 años libres de fallas (Lawrence et al, 2015). Obviamente, las publicaciones acerca de fallas en Sistemas ATR, también disminuyeron. Fue hasta el año 2015, cuando se presentaron nuevos problemas dieléctricos (Lawrence et al, 2015). Ahora, las fallas se presentaron en el lado línea de los autotransformadores. Se encontró que fueron ocasionadas por sobre tensiones generadas aleatoriamente por pre-igniciones múltiples, las cuales, a su vez, fueron ocasionadas al cerrar el interruptor principal del Sistema ATR, durante el proceso de arranque. Las sobre tensiones no ocasionaron rompimientos dieléctricos entre fases o de fase a tierra. Sin embargo, sus frecuencias fueron capaces de excitar una resonancia interna en el autotransformador, ocasionando una falla dieléctrica. Para resolver este problema, Lawrence et al recomendaron que también se instalen apartarrayos y supresores $\mathrm{RC}$, en el lado línea de los Sistemas ATR (Lawrence et al, 2015).

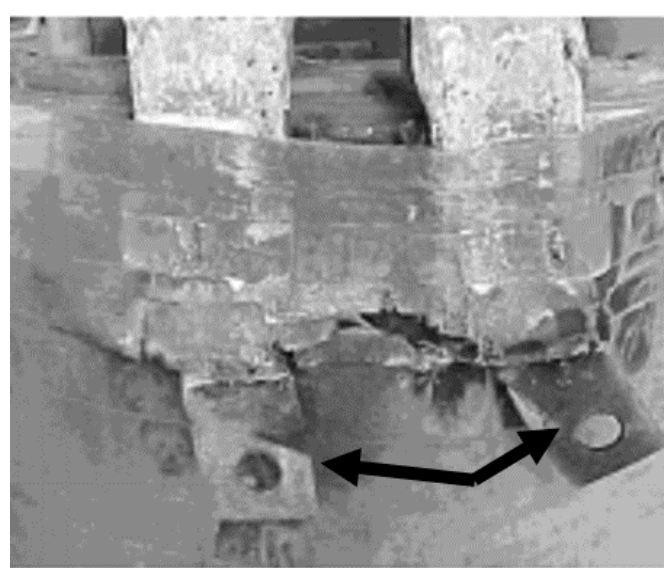

a) Falla entre las derivaciones del 0 y $50 \%$

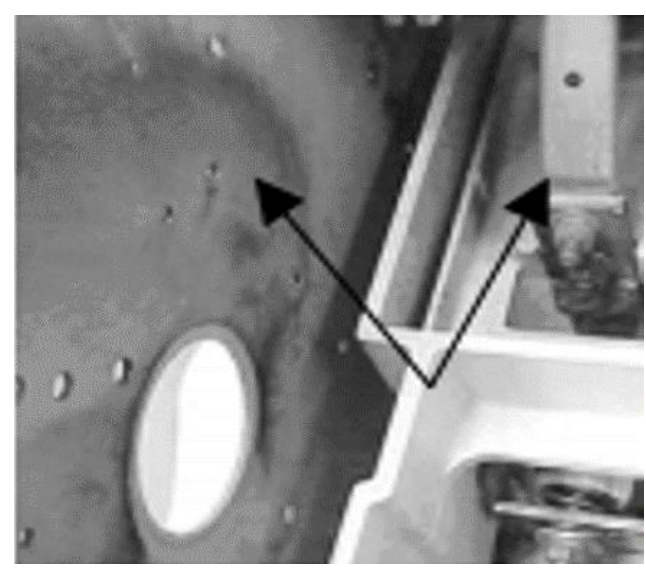

b) Arco eléctrico contra el gabinete

Fig. 1 Fallas en Sistemas ATR de media tensión, ocasionadas por sobretensiones en el lado neutro de los autotransformadores. Adaptada de Lawrence y Arthur, 2004. 
En este artículo, se presenta un mecanismo de falla, que también puede ocurrir en un Sistema ATR de media tensión, pero que es diferente a los descritos en los párrafos anteriores. A diferencia de las sobre tensiones en el lado neutro, ocasionadas por re-igniciones múltiples y escalación de tensiones, que se pueden presentar al abrir el interruptor del neutro, durante el proceso de arranque y a diferencia de las sobre tensiones en el lado línea, ocasionadas por pre-igniciones, que se pueden presentar al cerrar el interruptor principal durante el proceso de arranque, en este artículo, se discuten las sobre tensiones en el lado línea, ocasionadas por reigniciones múltiples y escalación de tensiones, que se pueden presentar al abrir el interruptor principal, durante el proceso de arranque. Este mecanismo se presentó a finales del año 2015 y a principios del año 2016, ocasionando fallas dieléctricas en tres Sistemas ATR de $13.8 \mathrm{kV}$, instalados en plantas petroquímicas mexicanas. En dos de las fallas, se encontraron evidencias de arcos eléctricos en el lado línea de los autotransformadores, ocasionados por una sobre tensión entre fases. En la tercera falla, se encontró un corto circuito en los devanados del autotransformador, en el lado línea, el cual fue ocasionado por una resonancia interna, la cual, a su vez, fue excitada por las frecuencias de re-igniciones múltiples, en el lado línea.

La apertura del interruptor principal de los Sistemas ATR, no forma parte del proceso de arranque del motor, por lo que puede ser considerada como una condición anormal de operación. Sin embargo, esta situación se presenta, si en el proceso de arranque, ocurre una falla, la cual puede accionar una protección y ésta a su vez, puede accionar la apertura del interruptor principal. Por ejemplo, una secuencia de arranque equivocada, en el sistema de control, puede generar una falla trifásica. Esto puede accionar una protección y disparar el interruptor principal. También una condición de rotor bloqueado o una secuencia incompleta de arranque, en un motor síncrono, entre otros casos, pueden accionar una protección y ocasionar el disparo del interruptor principal, durante el proceso de arranque.

A diferencia de las sobre tensiones por pre-igniciones en el lado línea, que solo pueden dañar a los autotransformadores por resonancias internas, las sobre tensiones por re-igniciones múltiples y escalación de tensiones, pueden ocasionar fallas entre fases o de fase a tierra, en el lado línea del sistema ATR. Además, pueden dañar a los autotransformadores por resonancias internas. También pueden ocasionar la falla del interruptor principal o una falla dieléctrica en cualquier otro equipo conectado al mismo bus de alimentación (Gajjar et al, 2013). Afortunadamente, la instalación de apartarrayos y supresores RC, en el lado línea, recomendados por Lawrence et al, para la protección contra sobre tensiones por pre-igniciones múltiples (Lawrence et al, 2015), también pueden minimizar las sobre tensiones por re-igniciones múltiples y escalación de tensiones.

Como se comentó anteriormente, la instalación de apartarrayos y supresores de transitorios $\mathrm{RC}$ en el neutro de los Sistemas ATR, se llevó a cabo en un período de 4 años aproximadamente. En consecuencia, la instalación de apartarrayos y supresores de transitorios RC, en el lado línea, puede demorar un período similar. No obstante, las experiencias reportadas en este artículo, pueden ser una justificación adicional, para que los usuarios de Sistemas ATR se decidan a instalar apartarrayos y supresores de transitorios RC, en el lado línea. El costo de estos accesorios es relativamente reducido con respecto al costo del autotransformador y con respecto a los costos y consecuencias técnicas generadas por una falla en el lado línea, ya sea en el Sistema ATR, en el interruptor principal o en algún otro equipo conectado a este mismo bus.

\section{ARREGLO TÍPICO DE LOS SISTEMAS ATR DE 13.8 kV}

En la Fig. 2 se muestra el diagrama típico de los Sistemas ATR, instalados en plantas petroquímicas mexicanas, para efectuar el arranque de motores síncronos de gran capacidad.

El proceso de arranque se lleva a cabo en cinco etapas, coordinadas por un sistema de control automático. En la Etapa Uno, se oprime el botón de arranque y a los $3 \mathrm{~s}$, se cierra el interruptor 3, conectando en estrella al neutro del autotransformador. A los $5 \mathrm{~s}$, se realiza la Etapa Dos, cerrando el interruptor principal (interruptor 1). Inmediatamente, el autotransformador se energiza al $100 \%$ de la tensión nominal, mientras que el motor se energiza al $80 \%$ de su tensión nominal. El arranque del motor al $80 \%$ de su tensión nominal, reduce la corriente de arranque.

La Etapa Tres, del proceso de arranque, se efectúa después de los $11 \mathrm{~s}$, cuando el motor se aproxima al 90\% de su velocidad nominal. En esta etapa, el control automático abre el interruptor del neutro (interruptor 3). En estas condiciones, el autotransformador se convierte en tres reactores monofásicos, que continúan limitando la corriente de arranque. A los $12 \mathrm{~s}$, se realiza la Etapa Cuatro, cerrando el interruptor del motor (interruptor 2). Esta maniobra deja fuera de servicio a los reactores monofásicos y efectúa la transición en la alimentación del motor, del 80 al $100 \%$ de la tensión nominal. Finalmente, a los $14 \mathrm{~s}$, cuando el motor ha alcanzado su velocidad nominal, se conecta la excitación del devanado de campo (Etapa Cinco). Cuando se desea sacar de servicio el motor, se oprime solamente el botón de "paro" y automáticamente se abren los interruptores 1 y 3. En la Fig. 2, también se observa la lista de las protecciones principales de los Sistemas ATR. 


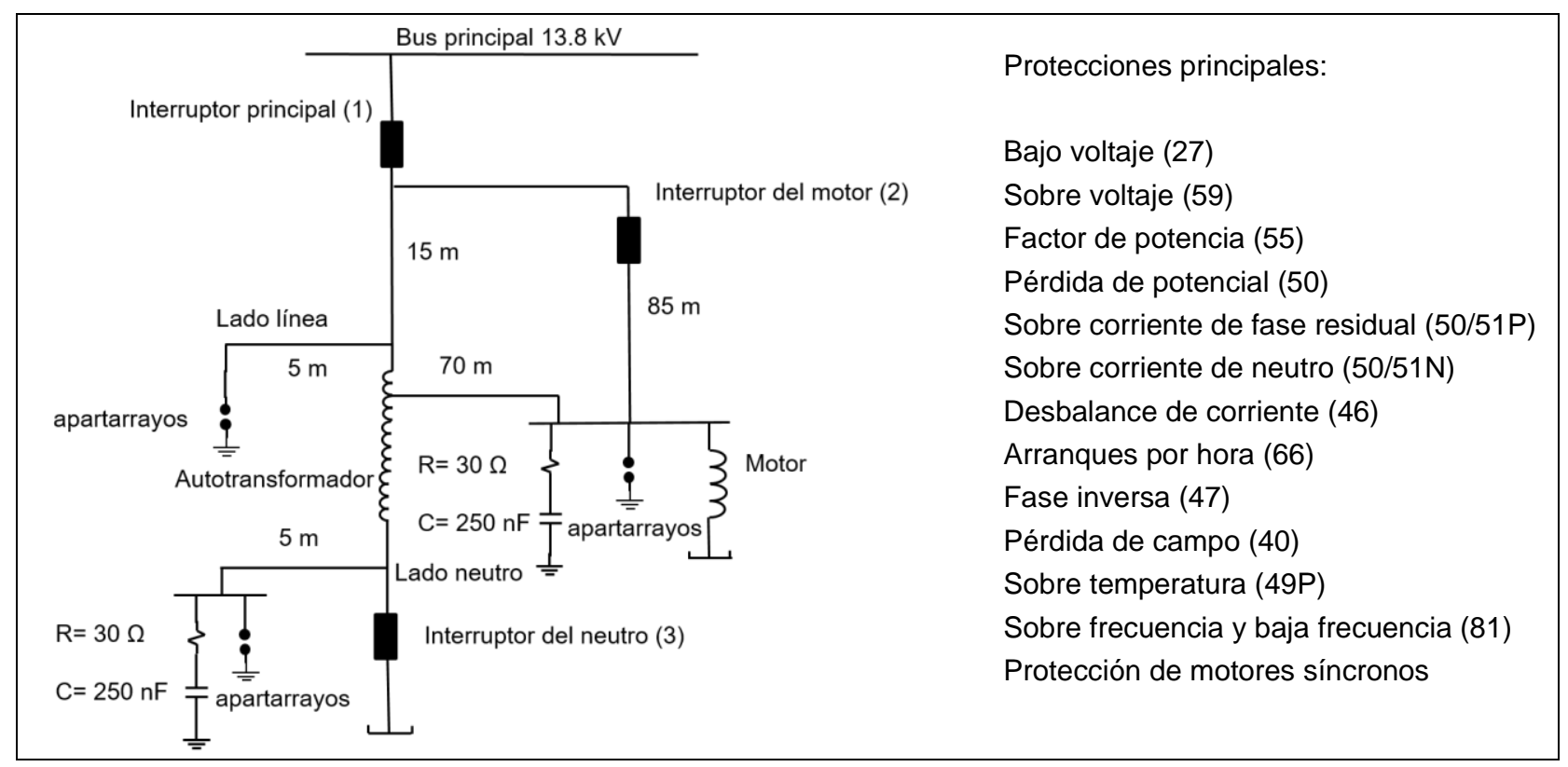

Fig. 2 Diagrama típico de un Sistema ATR de $13.8 \mathrm{kV}$, instalado en plantas petroquímicas mexicanas. Las conexiones se llevan a cabo con cable Clase $15 \mathrm{kV}$.

Se utilizan relevadores instantáneos de sobre corriente no direccional, para liberar las fallas entre fases y las fallas a tierra (relevadores $50 \mathrm{P}$ y $50 \mathrm{~N}$ respectivamente). Con respecto a la protección de motores síncronos, los relevadores 48 y 86 , son los que disparan directamente al interruptor principal, cuando el motor síncrono no ha alcanzado su velocidad síncrona o cuando el rotor se encuentra bloqueado. De acuerdo con las recomendaciones publicadas en la literatura especializada (Lawrence y Arthur, 2004; Vollet y De Metz Nobat, 2007; Emam, et al, 2008), para la protección contra las sobretensiones aleatorias generadas por la desconexión del neutro, todos los Sistemas ATR cuentan con apartarrayos y supresores de transitorios RC, conectados en el lado neutro del autotransformador y en las terminales del motor. Adicionalmente, se cuenta con apartarrayos en el lado línea de los autotransformadores. Todos los Sistemas ATR soportaron $35 \mathrm{kV}$ de CA durante un minuto y $95 \mathrm{kV}$ de tensión de impulso, durante las pruebas de fábrica.

\section{SOBRETENSIONES POR RE-ENCENDIDOS MÚLTIPLES}

Una inductancia almacena energía magnética cuando circula una corriente a través de ella. Si esta corriente se interrumpe, la energía almacenada se convierte en energía eléctrica y se descarga a través de la capacitancia del sistema, tal como se muestra en la Fig. 3.a (Stout y Bogh, 1998; Mueller y Saemann, 2011; Schoonenberg y Smeets, 2013). En estas condiciones, se genera una diferencia de potencial en las terminales de la inductancia, la cual es directamente proporcional a la rapidez con que cambia la corriente en el tiempo. La forma de onda de la tensión, será oscilatoria y su frecuencia será la frecuencia de resonancia de la inductancia y de la capacitancia del sistema (ver la Fig. 3.a). Por otro lado, cuando un interruptor de vacío abre sus contactos, se forma un arco eléctrico entre la separación de estos, a través del cual, la corriente sigue circulando. Cuando la corriente de un polo alcanza un bajo nivel, el arco se vuelve inestable y tiende a desaparecer. En consecuencia, la corriente se interrumpe antes de su cruce natural por cero. Este parámetro se denomina corriente cortada ( $\mathrm{i}_{\mathrm{ch}}$ ), ver la Fig. 3.a. Esta corriente amplifica la tensión en las terminales de la inductancia (Umax), tal como se muestra en la ecuación (1), donde L y C son la inductancia y la capacitancia del sistema.

La corriente cortada (ich) depende principalmente del material de los contactos del interruptor de vacío (Schoonenberg y Smeets, 2013). En la década de los setentas, los contactos eran fabricados solamente con cobre y la corriente cortada tenía valores hasta de $25 \mathrm{~A}$. En la década de los ochentas, se empezaron a fabricar contactos con una aleación de cobre-bromo, los cuales redujeron la corriente cortada hasta valores de $5 \mathrm{~A}$.

$$
\mathrm{U}_{\max }=\left(\sqrt{\frac{\mathrm{L}}{\mathrm{C}}}\right)\left(\mathrm{i}_{\mathrm{ch}}\right)
$$




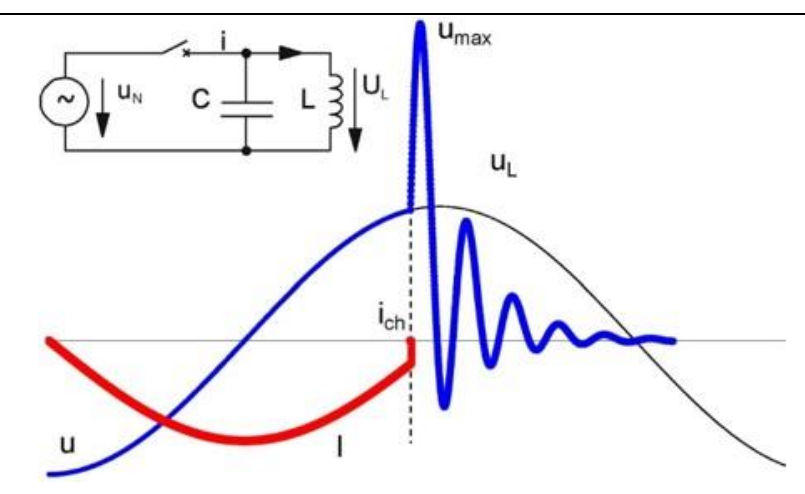

a.- Sobretensión por desconexión de una inductancia

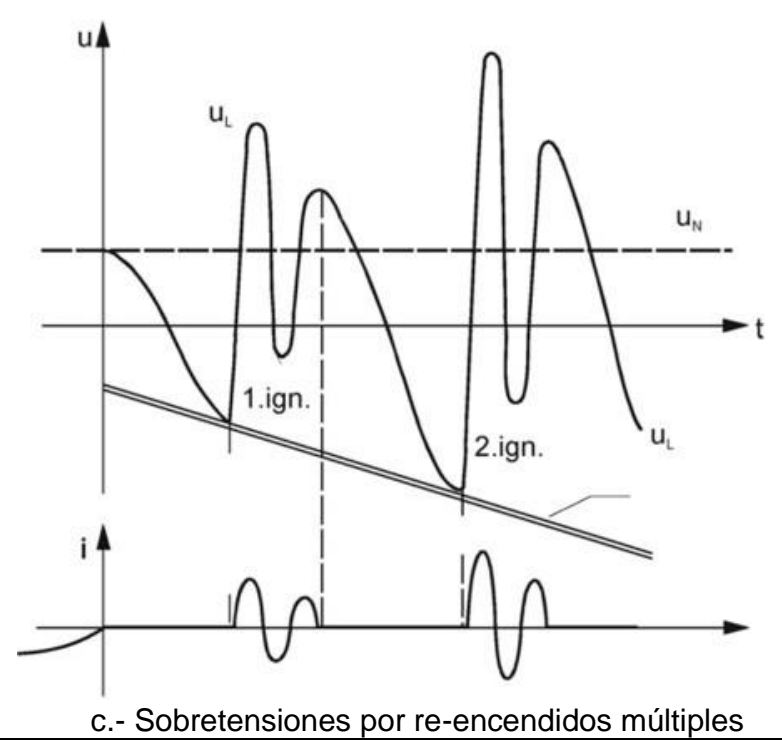

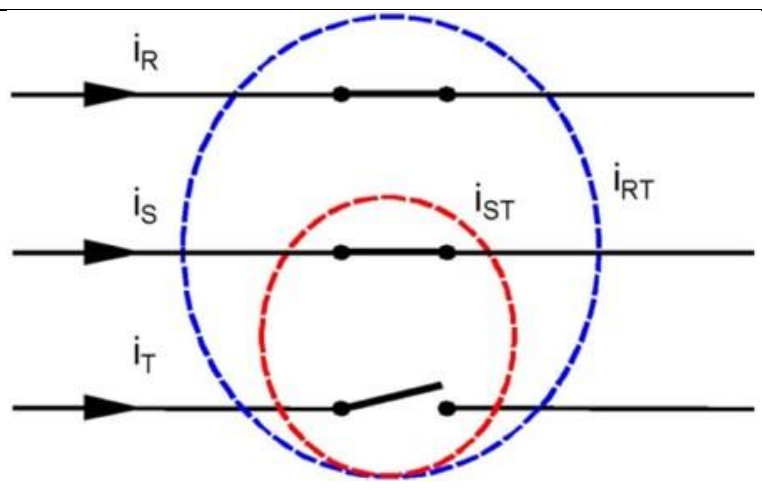

b.- Acoplamiento de la corriente de alta frecuencia

$U_{N}$ Tensión nominal del sistema I Corriente nominal del sistema UL Tensión en la inductancia ich Corriente cortada

$\cup_{\max }$ sobre tensión en las terminales de la inductancia $\mathrm{i}_{\mathrm{R}}$ Corriente de $60 \mathrm{~Hz}$ en la Fase $\mathrm{R}$

is Corriente de $60 \mathrm{~Hz}$ en la Fase $\mathrm{S}$ іт Corriente de $60 \mathrm{~Hz}$ en la Fase $\mathrm{T}$

ist Corriente de alta frecuencia acoplada entre las fases $S$ y $T$

$I_{R T}$ Corriente de alta frecuencia acoplada entre las fases $R$ y $T$

1.ign. ignición o re-encendido 1

2.ign. ignición o re-encendido 2

Fig. 3 Sobretensiones por desconexión de una inductancia y por re-encendidos múltiples. Adaptada de Mueller y Saemann, 2011.

Si la sobre tensión $\left(\cup_{\max }\right)$, genera una diferencia de potencial que supera la rigidez dieléctrica entre la separación de los contactos, ocurrirá un re-encendido del arco eléctrico, durante el proceso de apertura del interruptor (Schoonenberg y Smeets, 2013). Por ejemplo, si el re-encendido ocurre en la Fase T de la Fig. 3b, éste generará una corriente de alta frecuencia, que se acoplará capacitivamente en las otras dos fases, cuyos contactos van iniciando su apertura. Si los polos de las Fases $\mathrm{R}$ y $\mathrm{S}$ terminan de interrumpir la corriente de alta frecuencia cerca de uno de sus cruces por cero, se formará una nueva corriente cortada, denominada corriente cortada virtual, en las Fases R y S. La corriente virtual es mayor con respecto a la corriente cortada normal, por lo que ocasionará una nueva sobre tensión, en las Fases $\mathrm{R}$ y $\mathrm{S}$, pero ahora amplificada (escalación de tensiones). Por otro lado, la diferencia de potencial entre las Fases R y S puede ser hasta dos veces la sobre tensión de fase a tierra de estas dos fases. En la Fig. 3.c se muestra una secuencia de reencendidos múltiples y las sobretensiones amplificadas por escalación de tensiones (Mueller y Saeman, 2011).

Los re-encendidos múltiples y la escalación de tensiones, son fenómenos aleatorios. Esto indica que no se presentan en todas las desconexiones de un interruptor de vacío (Schoonenberg y Smeets, 2013). Su aparición depende principalmente de la inductancia y de la capacitancia del sistema, de los materiales de los contactos y del punto de la forma de onda de la corriente donde se lleve a cabo la desconexión del interruptor. Sin embargo, una vez que estos fenómenos se presentan, solamente se detendrán hasta que la fuerza dieléctrica entre la separación de los contactos logre soportar la diferencia de potencial entre ellos. En su defecto, se detendrán hasta que las sobretensiones ocasionen una falla dieléctrica.

En los Sistemas ATR de media tensión, se han registrado sobretensiones ocasionadas por re-encendidos múltiples y escalación de tensiones, en el lado neutro del autotransformador, con niveles hasta de $70 \mathrm{kV}$, con una frecuencia de oscilación de $850 \mathrm{kHz}$ y con una duración menor de 2 ms (Emam et al, 2008). 


\section{FALLA NÚMERO UNO}

El Sistema ATR, que presentó la Falla Número Uno, arrancaba un motor síncrono de 3500 HP. Este sistema fue puesto en servicio en el año 2002 y contaba con un autotransformador encapsulado en resina epóxica. En el período del año 2002 al 2007, el sistema presentó solamente una falla, en el lado del neutro del autotransformador. La falla ocurrió al desconectar el interruptor del neutro, durante el proceso de arranque del motor (Etapa Tres del proceso de arranque). Al abrir el interruptor, se presentó una sobre tensión que ocasionó un arco eléctrico entre el lado del neutro del autotransformador y el gabinete metálico, conectado a tierra.

En el año 2008, el Sistema ATR fue acondicionado con apartarrayos y supresores de transitorios RC, en el lado neutro del autotransformador, tal como se muestra en la Fig. 2. Con esta medida correctiva, se logró eliminar las fallas en el lado neutro del autotransformador. Sin embargo, después de un período de 7 años consecutivos, con arranques libres de fallas, se presentó una falla a principios del año 2016. Justo antes de la falla, el motor síncrono estaba operando normalmente y salió de servicio para poder realizar unos trabajos de mantenimiento, en el tren de producción. Entre los trabajos realizados, se efectuó la inspección y limpieza general de los componentes del Sistema ATR. Al terminar el mantenimiento, se programó nuevamente la puesta en servicio del motor. Sin embargo, cuando se realizaba el proceso de arranque, se presentó una falla en el Sistema ATR, que sacó de servicio todas las cargas conectadas al bus de alimentación.

Con la medición de la resistencia de aislamiento, todos los equipos del sistema arrancador-motor se encontraron en buenas condiciones dieléctricas. Solamente se encontró un detalle anormal, en el lado línea del autotransformador. En las esquinas de los bornes de conexión, entre los cables procedentes del interruptor principal y los devanados del autotransformador, en las Fases 1 y 2, se encontraron evidencias de erosión, ocasionada por un arco eléctrico. Las evidencias se muestran encerradas con elipses de color blanco, en la Fig. 4. Es importante señalar que estas evidencias no fueron identificadas durante los trabajos de mantenimiento, realizados justo antes de la falla. Estas evidencias indicaron que posiblemente había ocurrido un arco eléctrico entre las Fases 1 y 2, en el lado línea del autotransformador. En la Fig. 4, también se muestra la posible trayectoria del arco eléctrico, con flechas blancas.

En el esquema de protecciones, se encontró que el relevador 50P se había activado, abriendo el interruptor principal, durante el proceso de arranque. El relevador 50P no tenía la capacidad para registrar las formas de onda generadas por la falla. Sin embargo, el nivel de la corriente de falla alcanzó el umbral de disparo de un relevador 51P, que se encontraba instalado en un interruptor "aguas arriba". Este relevador sí registró las formas de onda durante la falla. En la Fig. 5, se muestra la ubicación de los relevadores 50P y 51P y en la Fig. 6, se muestran las formas de onda registradas por el relevador 51P.

Inicialmente, se consideró que el arco eléctrico entre las Fases 1 y 2 había sido el origen de la falla del Sistema ATR. Sin embargo, de acuerdo con las formas de onda de la Fig. 6, se observa que las tres fases registraron una sobre corriente del orden de $10 \mathrm{kA}$. Esto indicó que la falla no fue ocasionada por un arco eléctrico entre dos fases, sino por un corto circuito trifásico. En la Fig. 6, también se observa que el relevador 51P no logró disparar, debido al retardo de tiempo que tenía programado. No obstante, el relevador 51P dejó de registrar la corriente de falla, en cuanto la protección 50P, abrió el interruptor principal del Sistema ATR.

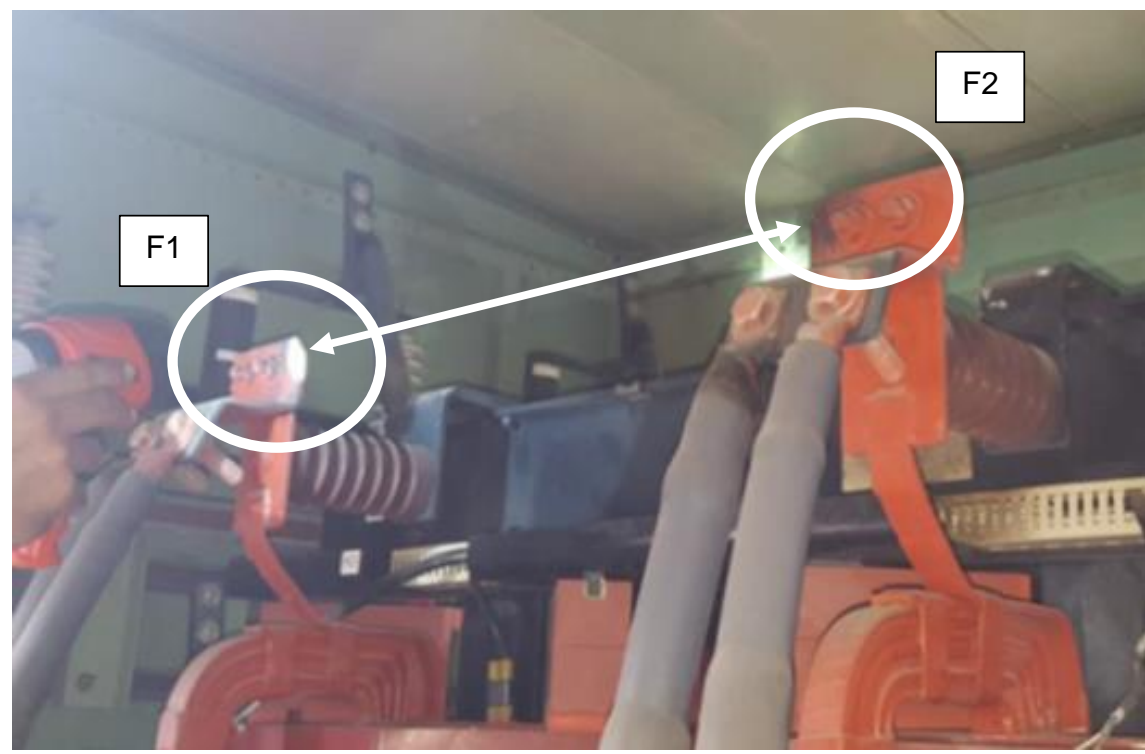

Fig. 4 Erosión eléctrica en los bornes de conexión y trayectoria posible de un arco eléctrico entre las Fases 1 y 2 , en el lado línea del autotransformador de un Sistema ATR 


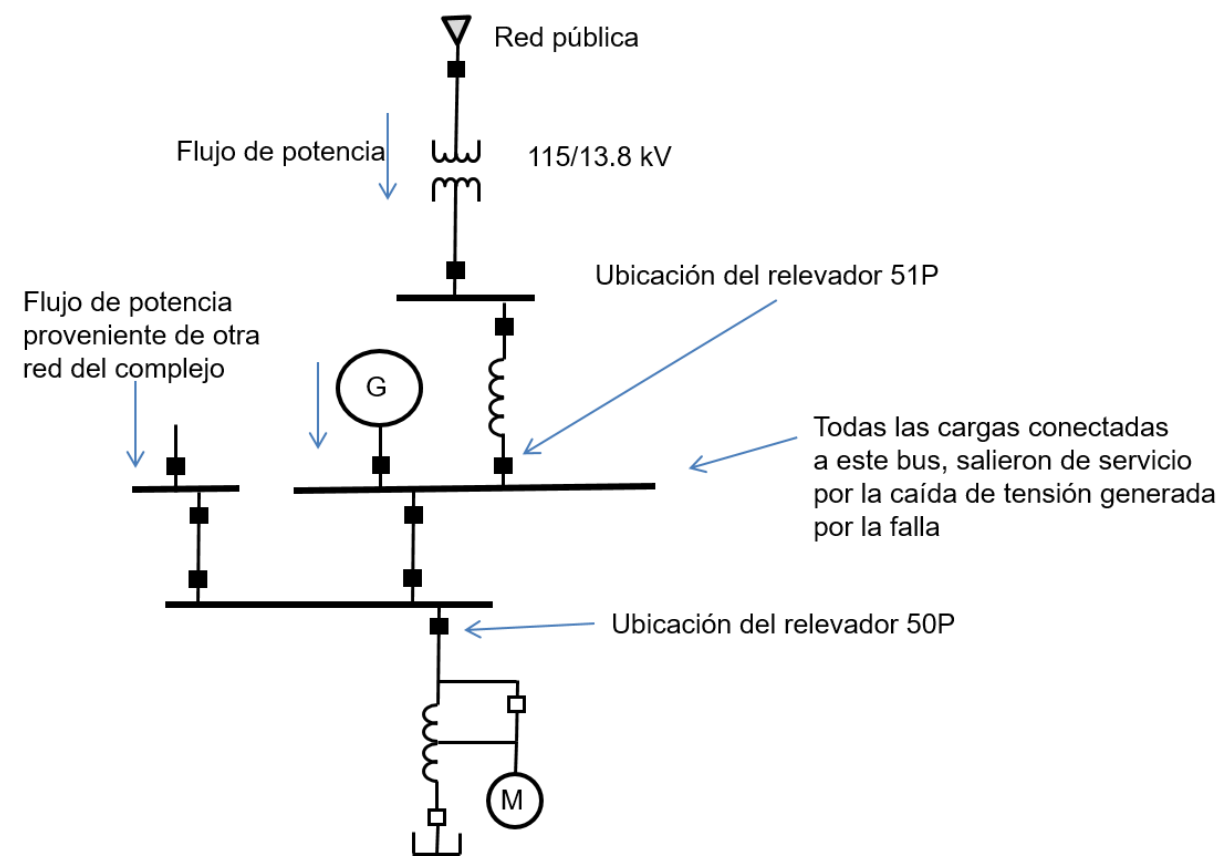

Fig. 5 Ubicación de los relevadores 50P y 51P con respecto al Sistema ATR

En la Fig. 6, también se observa que el corto circuito trifásico generó una caída de tensión severa en el bus de alimentación, durante el evento del corto circuito trifásico. Esto ocasionó la salida de servicio de todas las cargas conectadas a este bus. Al revisar el sistema de control, se encontró que la secuencia de arranque se realizó en forma equivocada. Primero, se cerraron el interruptor del motor y el interruptor principal del Sistema ATR. En estas condiciones, el motor arrancó a tensión plena. El sistema soportó esta condición transitoria, debido a que contaba con el respaldo de la red pública.

Como el interruptor principal cerró primero y el interruptor del neutro se encontraba abierto, las terminales del neutro quedaron desconectadas, tal como se muestra en la Fig. 7. Estas terminales adquirieron un alto potencial, el cual fue generado por el autotransformador, operando como un transformador elevador. En estas condiciones, al cerrar el interruptor del neutro, se generó un corto circuito trifásico. Esto accionó la protección 50P y consecuentemente, la apertura del interruptor principal. Entonces, ¿cómo se generó la erosión eléctrica de los bornes de conexión de las Fases 1 y 2? Para superar la rigidez dieléctrica entre las terminales de línea de las fases F1 y F2 tuvo que presentarse una diferencia de potencial mayor a la que se presentó durante las pruebas en fábrica ( $95 \mathrm{kV}$ de la prueba de impulso). Durante el proceso de arranque, no estaban ocurriendo descargas atmosféricas, por lo tanto, la sobre tensión tuvo que ser generada por la maniobra de los interruptores.

En este caso, la sobre tensión no pudo ser generada por el cierre del interruptor principal, al iniciar el proceso de arranque, debido a que el motor arrancó a tensión plena sin ninguna falla. Tampoco pudo ser generada por la apertura del interruptor del neutro, debido a que el Sistema ATR nunca llegó a esta etapa del proceso de arranque. Así que la única maniobra que pudo ocasionar la sobre tensión, es la apertura del interruptor principal, accionada por el disparo de la protección 50P.

Cuando el interruptor principal del Sistema ATR se abre para detener el funcionamiento del motor, se interrumpe como máximo la corriente nominal del motor. Por otro lado, éste se comporta como un generador síncrono y tiende a mantener la tensión en terminales, de manera que no se presenta ninguna sobre tensión por la desconexión de la inductancia. En cambio, cuando el interruptor principal se abre durante un corto circuito trifásico, justo en el proceso de arranque del motor, se interrumpe una corriente transitoria considerable y la fuerza contra electromotriz generada por el motor, no se puede oponer a una sobre tensión en las terminales de la inductancia. Por lo tanto, se puede generar una sobre tensión en el lado línea del autotransformador. Posteriormente, esta sobre tensión pudo ocasionar el fenómeno de re-encendidos múltiples, con escalación de tensiones.

A diferencia de la prueba de impulso de $95 \mathrm{kV}$, la cual se aplica entre línea y tierra, en cada fase individualmente, con las otras dos fases conectadas a tierra, las sobretensiones por los fenómenos de reencendidos múltiples y escalación de tensiones se presentan en dos fases prácticamente en forma simultánea, tal como se describió en la sección anterior. 


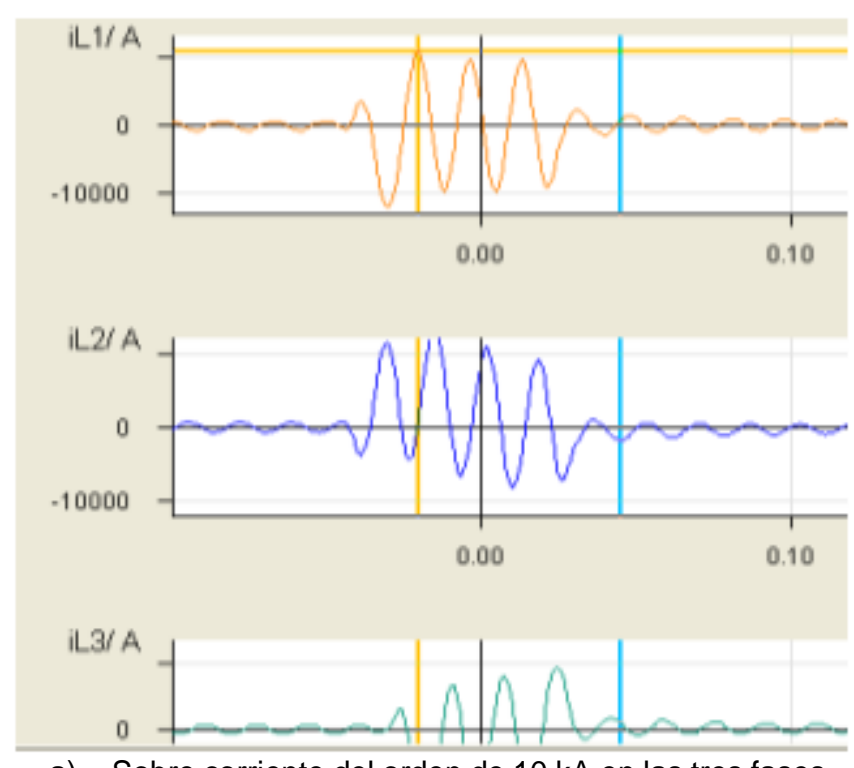

a) Sobre corriente del orden de $10 \mathrm{kA}$ en las tres fases

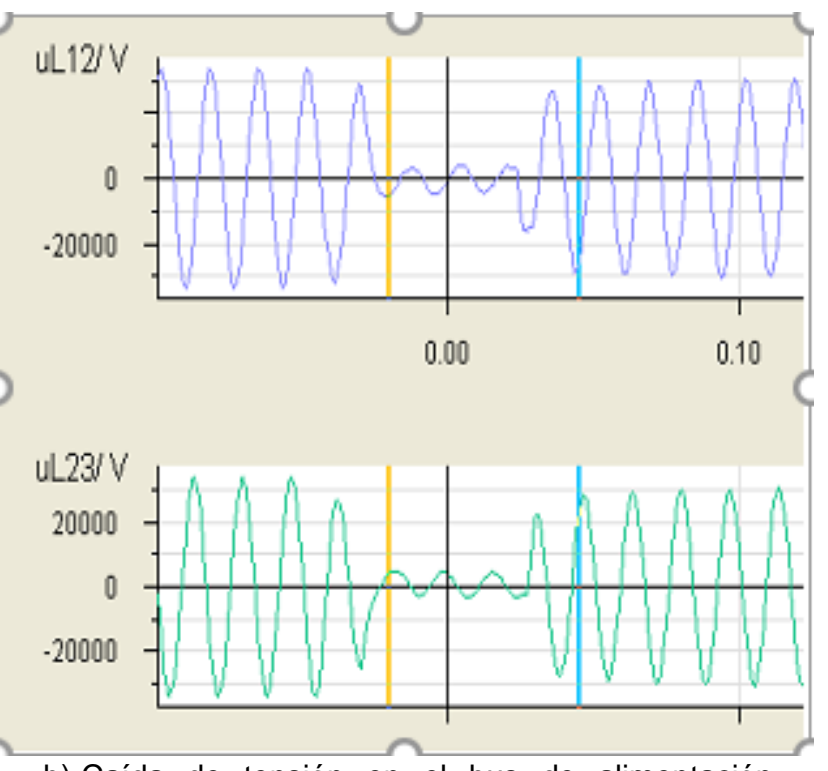

b) Caída de tensión en el bus de alimentación, generada por el corto circuito trifásico en el Sistema ATR

Fig. 6 Formas de onda registradas por un relevador 51P, instalado en un interruptor "aguas arriba", con respecto al interruptor principal del Sistema ATR

Suponiendo que las sobretensiones en las fases F1 y F2, fueran de $70 \mathrm{kV}$ con respecto a tierra, como se ha reportado en la literatura especializada (Emam et al, 2008), la diferencia de potencial entre las dos fases podría ser hasta de $140 \mathrm{kV}$, lo cual superaría el nivel de aislamiento del Sistema ATR y ocasionaría el arco eléctrico entre las Fases F1 y F2.

Finalmente, es importante señalar que el Sistema ATR, contaba con apartarrayos, instalados en el lado línea del autotransformador. Sin embargo, tal como lo indican Telander y Ouahdi, los apartarrayos no son suficientes para proteger a los Sistemas ATR, contra las sobretensiones por re-encendidos múltiples y escalación de tensiones (Telander et al, 1987; Ouahdi, 1991). Los apartarrayos pudieron reducir las sobretensiones iniciales, durante la apertura del interruptor principal, pero no pudieron modificar la frecuencia de éstas. Esto permitió la formación de más sobretensiones por re-encendidos múltiples y la escalación de tensiones, las cuales terminaron por generar el arco eléctrico entre las Fases F1 y F2. Para minimizar las posibilidades de que se formen re-encendidos en el lado línea de los autotransformadores, es necesario que se instale en esta zona, una combinación de apartarrayos y supresores de transitorios RC, tal como se instalaron en el lado neutro del autotransformador. Esto es lo recomendado por Lawrence et al (2015).

\section{FALLA NÚMERO DOS}

Esta falla se presentó durante la puesta en servicio de un Sistema ATR nuevo, que arrancaba un motor síncrono de $2500 \mathrm{HP}$, al $80 \%$ de su tensión nominal. El Sistema ATR contaba con el diagrama unifilar que se muestra en la Fig. 2 y con un autotransformador del tipo seco. En la Fig. 8.a se muestra una fotografía de la placa, que contiene el diagrama de conexiones del autotransformador. El proceso de la Falla Número Dos fue similar al proceso de la Falla Número Uno. Una falla en el sistema de control permitió que el motor arrancara a tensión plena y las terminales del neutro quedaron desconectadas, con un alto potencial. Posteriormente, al cerrar el interruptor del neutro, se generó un corto circuito trifásico, el cual ocasionó una caída de tensión considerable, que sacó de servicio a todas las cargas conectadas en el bus de alimentación. El corto circuito trifásico también accionó la protección 50P y consecuentemente, la apertura del interruptor principal. Después de la falla, todos los equipos del sistema arrancador-motor se encontraron en buenas condiciones dieléctricas. A diferencia de la Falla Número Uno, la Falla Número Dos dejó una evidencia más clara del arco eléctrico que se formó entre terminales con diferente nivel de tensión. En la Fig. 8.b, se muestran los residuos de carbón, que fueron generados por un arco eléctrico entre las terminales $\mathrm{H} 1$ y las terminales $\mathrm{H} 0$.

Durante el proceso de arranque del motor, no estaban ocurriendo descargas atmosféricas, de tal manera que para superar la rigidez dieléctrica entre las terminales $\mathrm{H} 0$ y $\mathrm{H} 1$ tuvo que presentarse una sobre tensión generada por la apertura del interruptor principal, al interrumpir la corriente de corto circuito. La sobre tensión, tuvo que generar el fenómeno de re-igniciones múltiples con escalación de tensiones, alcanzando una magnitud que superó los niveles de aislamiento, entre las terminales $\mathrm{H} 1$ y las terminales $\mathrm{H} 0$. 


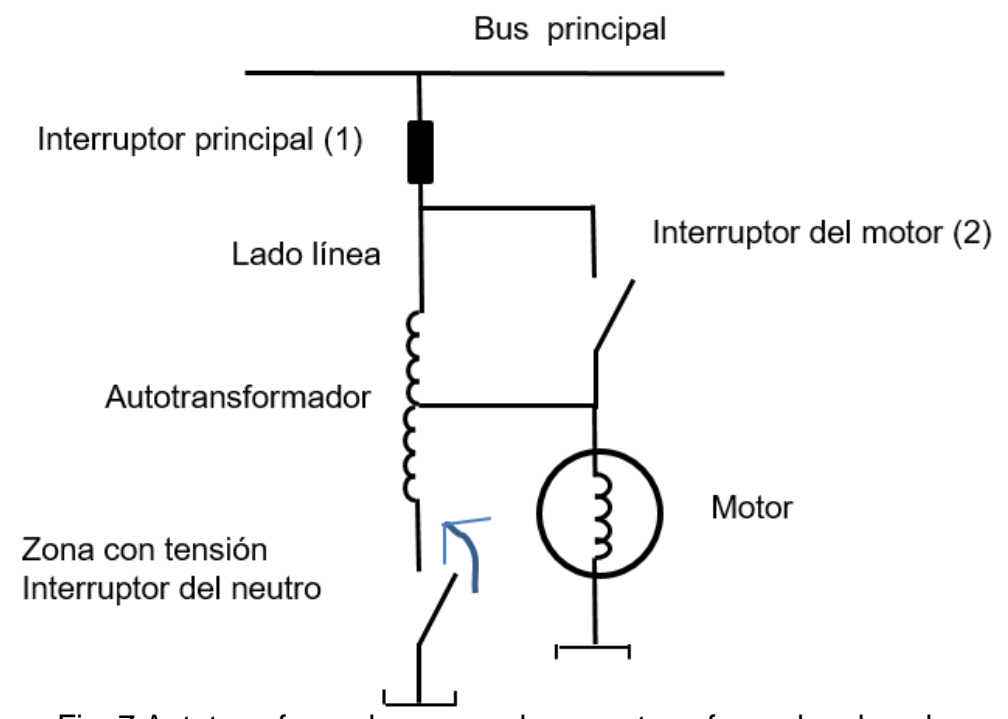

Fig. 7 Autotransformador operando como transformador elevador

\section{FALLA NÚMERO TRES}

Esta falla se presentó en el año 2016, en un Sistema ATR que arrancaba un motor síncrono de $4200 \mathrm{HP}$. El Sistema ATR tenía 6 años de antigüedad, contaba con el arreglo indicado por la Fig. 1 y con un autotransformador con aislamiento del tipo seco. En el historial de servicio del Sistema ATR, se encontró que el equipo había presentado problemas operativos, en forma aleatoria.

En algunos casos, el Sistema ATR arrancaba el motor sin mayores problemas. Sin embargo, en otros casos, el motor comenzaba a zumbar, pero no lograba acelerar. La protección de secuencia incompleta detectaba que el motor no había alcanzado su velocidad síncrona y accionaba la apertura del interruptor principal del Sistema ATR, interrumpiendo el proceso de arranque. Posteriormente, se tenía que realizar un segundo intento de arranque y el motor lograba alcanzar su velocidad síncrona, sin mayores problemas.

El día de la falla, la protección de secuencia incompleta interrumpió el primer intento de arranque a los $9 \mathrm{~s}$. Al efectuar el segundo intento de arranque, se activó la protección $50 \mathrm{~N}$, la cual disparó el interruptor principal. Al revisar los equipos del Sistema ATR, se encontró una falla en la Fase 2 del autotransformador, tal como se muestra en la Fig. 9. Cada bobina por fase del autotransformador, contaba con cinco secciones. El corto circuito se presentó en la Sección 1, cerca de la terminal de inicio de línea y evolucionó hacia una falla a tierra. Se consideró que la falla pudo ser ocasionada por uno de los siguientes mecanismos: (a) por una sobre tensión transitoria que ingresó al devanado del autotransformador, (b) por una resonancia interna excitada por la frecuencia de pre-igniciones múltiples o (c) por una resonancia interna excitada por la frecuencia de reencendidos múltiples y escalación de tensiones.

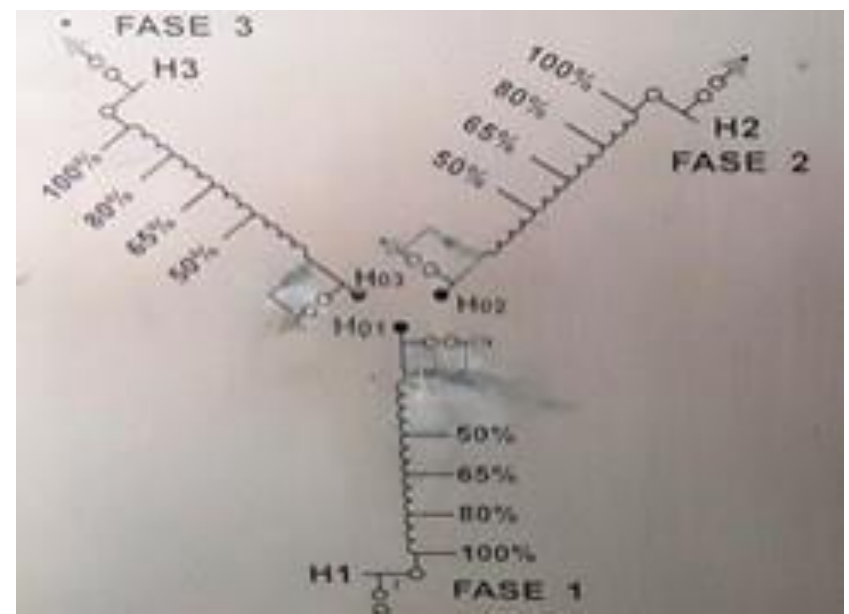

a) Diagrama de conexiones de los devanados del autotransformador

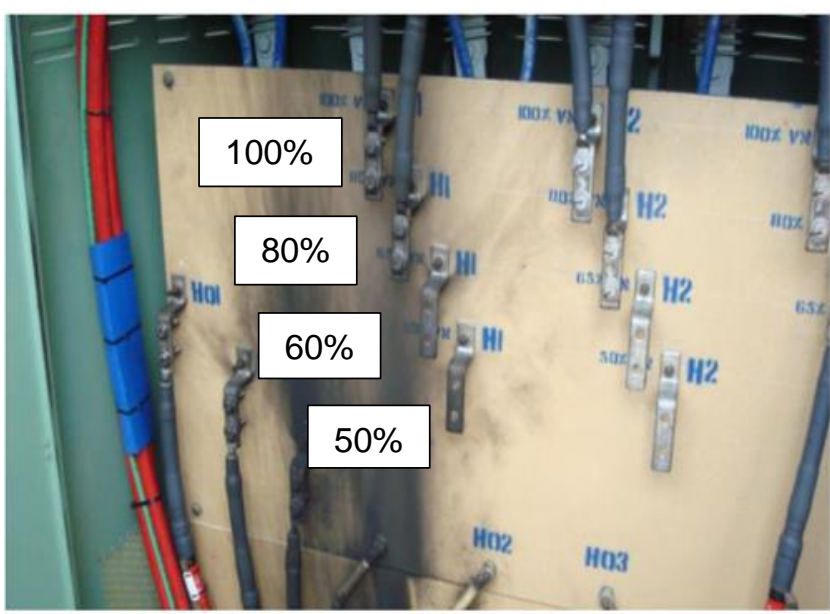

b) Panel frontal de conexiones del autotransformador, con evidencia de arco eléctrico

Fig. 8 Falla en un autotransformador durante el arranque de un Sistema ATR, ocasionada por una secuencia de arranque equivocada en el sistema de control 
Las sobretensiones del tipo onda viajera, con frentes de onda del orden de los microsegundos, que pueden arribar a los autotransformadores de los Sistemas ATR, pueden ser generadas por una descarga atmosférica. Estas sobretensiones someten a las primeras vueltas de los devanados a los más altos gradientes de tensión. En fábrica, el Sistema ATR bajo estudio, soportó $95 \mathrm{kV}$ de tensión de impulso (con una forma de onda de 1.2 x $50 \mu \mathrm{s}$ ). En este caso, la falla se ubica en la Sección 1, cerca del inicio de línea. Sin embargo, como en el momento de la falla, no había ninguna tormenta eléctrica, se descartó la posibilidad de que la falla hubiese sido ocasionada por este mecanismo.

Las pre-igniciones múltiples, que se presentan cuando se van cerrando los contactos de un interruptor de vacío, antes de que se establezca el contacto galvánico final, pueden ocasionar sobretensiones del tipo onda viajera. Al arribar a la carga, una parte de la sobre tensión se refracta y otra se refleja. Esto puede generar la superposición de ondas viajeras. Sin embargo, se ha reportado en la literatura especializada, que la magnitud de este tipo de sobretensiones normalmente no supera el nivel de aislamiento (Schoonenberg y Smeets, 2013). Por lo tanto, la hipótesis de que la falla fue ocasionada por una sobre tensión transitoria que ingresó al devanado del autotransformador, quedó descartada.

La resonancia interna en transformadores es un fenómeno recientemente reportado en la literatura especializada (Mitra et al, 2011). Este fenómeno explica por qué ocurren fallas internas ocasionadas por sobretensiones, en los transformadores, aunque éstos se encuentren protegidos con apartarrayos, instalados en sus terminales de línea. En el caso de los Sistemas ATR, la resonancia interna, excitada por la frecuencia de sobretensiones, las cuales, a su vez, fueron ocasionadas por pre-igniciones múltiples, es el modo de falla recientemente reportado por Lawrence et al (Lawrence et al, 2015). En su artículo, Lawrence et al indicaron que la frecuencia natural de los autotransformadores de los Sistemas ATR típicamente se encuentra entre 5 $\mathrm{kHz}$ y unos cientos de kHz. En el ejemplo reportado, el autotransformador tenía una frecuencia de resonancia serie de $22 \mathrm{kHz}$ y ésta fue excitada por la frecuencia de $21.2 \mathrm{kHz}$, que se presentó en las pre-igniciones múltiples, que fueron ocasionadas al cerrar el interruptor principal del Sistema ATR. La resonancia generó una falla entre capas, que se ubicó entre la primera mitad y el final del devanado de una de las fases.

Otro estudio, recientemente reportado, indica que la ubicación de una falla generada por una resonancia interna, está relacionada con el valor de la frecuencia de resonancia (Florkowski y Furjal, 2009). Si la frecuencia de resonancia es del orden de algunos $\mathrm{kHz}$, la falla tenderá a presentarse cerca de la mitad del devanado, tal como ocurrió en el ejemplo reportado por Lawrence et al (Lawrence et al, 2015). En cambio, si la frecuencia de resonancia se desplaza hacia los $\mathrm{MHz}$, la falla tenderá a presentarse cerca del inicio del devanado (Florkowski y Furjal, 2009).

En el caso de la Fig. 9, la falla se encontró en la Sección 1 del devanado, cerca de la terminal de línea, por lo que se estima que la frecuencia de resonancia pudo tener un valor tendiente hacia los $\mathrm{MHz}$. Por otro lado, Emam et al, reportaron una frecuencia del orden de $850 \mathrm{kHz}$, en sobretensiones ocasionadas por reencendidos múltiples y escalación de tensiones (Emam et al, 2008). Esto muestra que la frecuencia de las sobretensiones ocasionadas por estos fenómenos tiene una tendencia hacia los MHz.

De acuerdo con lo anterior, la Falla Número Tres, al localizarse cerca de la terminal de línea, pudo ser causada por una frecuencia tendiente hacia los $\mathrm{MHz}$, ocasionada por una sobre tensión por re-encendidos múltiples y escalación de tensiones, generada por la apertura del interruptor principal, durante el proceso de arranque del motor.

Los apartarrayos instalados en el lado línea del autotransformador, no cortaron la sobre tensión, porque esta no arribó en forma de onda viajera, sino que se presentó internamente, en los devanados del autotransformador. También es importante mencionar que una resonancia interna no necesariamente ocasiona una falla, en forma instantánea. Una falla, también puede ser ocasionada por el efecto acumulado, de un conjunto de sobretensiones generadas por resonancias internas, las cuales van deteriorando el aislamiento (Lawrence et al, 2015). En este caso, el Sistema ATR había sido sometido repetidamente a interrupciones del proceso de arranque, por el accionamiento de la protección de secuencia incompleta. Esto pudo ocasionar sobre tensiones por resonancias internas, al inicio de los devanados del autotransformador. El efecto acumulativo de estas sobretensiones pudo deteriorar el sistema aislante del autotransformador, hasta ocasionar una falla.

Para minimizar la posibilidad de que se formen re-encendidos múltiples y escalación de tensiones, en el lado línea de los Sistemas ATR, al abrir el interruptor principal durante el proceso de arranque, es necesario que se instale, en esta zona, una combinación de apartarrayos y supresores de transitorios RC. La efectividad y rentabilidad de esta medida correctiva ya ha sido demostrada, cuando estos elementos se instalaron en el lado neutro de los Sistemas ATR. 


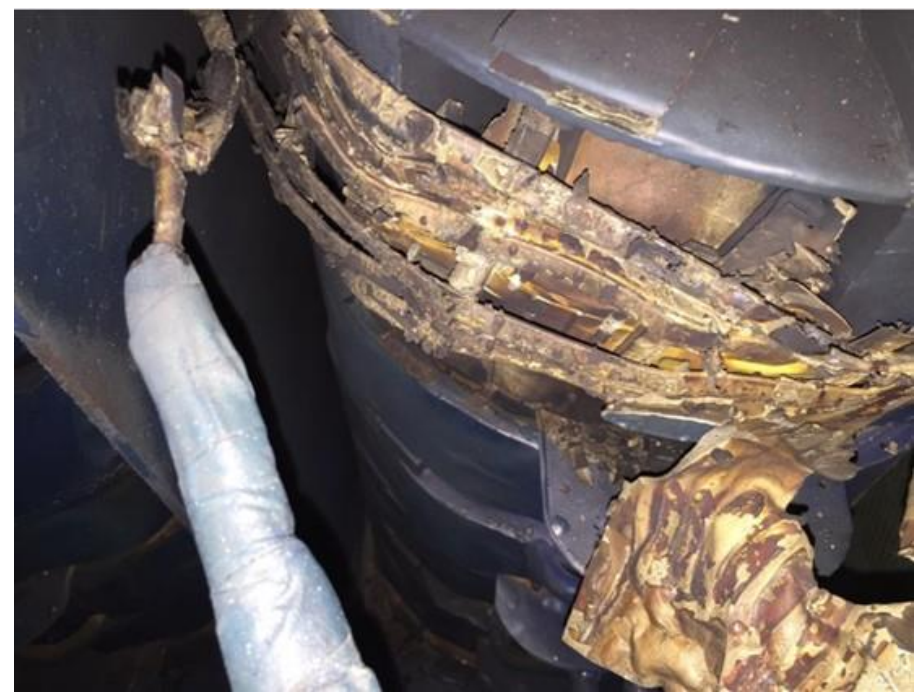

Fig. 9 Falla en un autotransformador ocasionada por una sobre tensión durante la interrupción del proceso de arranque

Es importante señalar que las sobre tensiones ocasionadas por re-encendidos múltiples y escalación de tensiones, también están siendo reportadas al abrir el interruptor principal para desconectar transformadores en vacío y para desconectar bancos de capacitores y compensadores estáticos de reactivos, en condiciones de falla (Shipp et al, 2012; Gajjar et al, 2013; Cisneros et al, 2015). Asimismo, se ha estado recomendando la instalación de apartarrayos y supresores de transitorios RC, en el lado línea, para la desconexión de transformadores en vacío (Shipp et al, 2012; Mardegan et al, 2016). Por otro lado, se han iniciado diferentes estudios para el desarrollo de mejores supresores de transitorios (Mejía et al, 2015).

\section{CONCLUSIONES}

La apertura del interruptor principal, es una maniobra que no se realiza durante el arranque de un Sistema ATR de media tensión. Sin embargo, esta situación se puede presentar, debido al accionamiento de alguna protección, la cual, a su vez, ocasione el disparo del interruptor principal. Esto puede generar sobretensiones aleatorias por re-encendidos múltiples y escalación de tensiones, en el lado línea del autotransformador. La magnitud de las sobretensiones puede ocasionar arcos eléctricos, principalmente entre fases. La frecuencia de las sobretensiones también puede excitar una resonancia interna en el autotransformador, ocasionando una falla en sus devanados. Finalmente, la escalación de tensiones también puede ocasionar una falla en el interruptor principal o puede incrementar la tensión en el bus y generar una falla en algún otro equipo, conectado a él.

Para proteger los Sistemas ATR, contra sobretensiones en el lado línea de los autotransformadores, se requiere que también se instale, en esta zona, una combinación de apartarrayos y supresores de transitorios $\mathrm{RC}$, tal como se han instalado en las terminales del motor y en el lado del neutro de los autotransformadores. Los apartarrayos reducen la magnitud de la sobre tensión y los supresores de transitorios RC modifican su frecuencia. Esto minimiza la posibilidad de que se generen re-encendidos y la escalación de tensiones. Con estas medidas, se podría incrementar la confiabilidad de los Sistemas ATR y ponerlos a competir nuevamente con otro tipo de arrancadores.

\section{REFERENCIAS}

Chaly, A.M. y Chalaya, A.T., The Influence of a Vacuum Circuit Breaker and Circuit Parameters on Switching Overvoltages Generated During Interruption of Starting Motors, International Symposium on Discharges and Electrical Insulation in Vacuum, July 21, 244-248, (1996)

Cisneros-Villalobos L., Oubram O., Limón-Mendoza, M., Aquino-Roblero F., Díaz-González L., Tecpoyotl-Torres M. y Montaño-Bautista C. I., Overvoltages in Static VAr Compensators due to Fault Breaker Opening, Simulation in ATP and the Actual Event, Revista Electrónica Nova Scientia, № 14 Vol. 7 (2), ISSN 2007 - 0705, 102 - 126, (2015)

Emam, S.E.A., Amer, A.H., Gaber, M., Protective Measures for Transient Overvoltages in Motors Starting by Autotransformers, doi: 10.1109/MEPCON.2008.4562397,12th International Middle East Power System Conference, MEPCON 2008, 503-510, (2008)

Florkowski M. y Furjal J., Terminal Based Recognition of Resonance Overvoltages in Transformer Windings, Proceedings of the $16^{\text {th }}$ International Symposium on High Voltage Engineering, Paper A-1, (2009) 
Gajjar G., Kulkarni A.M., Soman S.A., Interaction of Capacitor Bank Inrush Current Limiting Reactor and Medium Voltage Vacuum Circuit Breakers, International Conference on Power Systems Transients (IPST2013), Vancouver, Canada, July 18-20, (2013)

Hubbi, W. y Halak, M., Operational Problems with Large Induction Motors Connected to a Small Power System, Elsevier, Electric Power System Research, Volume 30, Issue 1, 57-61 (1994)

IEC 60470, High Voltage Switchgear Alternating Current Contactors and Contactor Based Motor Starters, (2000)

Lawrence, B.F. y Arthur, J.S., Medium Voltage Reduced Voltage Autotransformer Starter Failures -Explaining the Unexplained, IEEE Industry Applications Society, 51st Annual Petroleum and Chemical Industry Conference, September 13, 217-221, (2004)

Lawrence, B.F. y Thomas, F., Considerations in Medium Voltage Reduced Voltage Motor Starting the Good, the Bad and the Ugly, doi: 10.1109/PCICON.2007.4365792, IEEE Petroleum and Chemical Industry Technical Conference, PCIC, September 17, (2007)

Lawrence, B.F, Dave, S., Arthur, J.S., Paul, P.G., Steven, J., Medium Voltage Autotransformer Failures: Explaining the Unexplained - Continuation of the Story, IEEE Petroleum and Chemical Industry Committee Conference, PCIC 2015, doi: 10.1109/PCICON.2015.7435125, October 5-7, (2015)

Mardegan C.S., Shipp D.D., Melo L. A. R., Santana M.R., The Experience Acquired Sizing Snubbers to Mitigate Switching Transients in Industrial Power Systems, IEEE Transactions on Industry Applications, Vol. 52, Sept-Oct, (2016)

Mejía G. E., Muñoz N., y Cano J.B., Procedimiento Novedoso para el Diseño del Circuito Amortiguador RCD (Snubber RCD) y Deducción Matemática de las Ecuaciones, doi: 10.4067/S0718-07642015000200002, Información Tecnológica, Vol. 26(2), 117-128, (2015)

Mitra, P., De, A., Chakrabarti, A., Resonant Behavior of EHV Transformer Windings Under System Originated Oscillatory Transient Over Voltages, doi:10.1016/j.ijepes.2011.08.019, Electrical Power and Energy Systems 33, Elsevier, 1760-1766, (2011)

Mueller A. y Saemann D., Switching Phenomena in Medium Voltage Systems - Good Engineering Practice on the Application of Vacuum Circuit Breakers and Contactors, Petroleum and Chemical Industry Conference Europe Conference Procedings, PECIC Europe, (2011)

Ouahdi, D., The Protection of Induction Motors Against Switching Over Voltages Induced by Vacuum Circuit Breaker, Mining Technology, (1991)

Schoonenberg, G. y Smeets, R., Control of Inductive Load Switching Transients, doi: 10.1049/cp.2013.1132, 22nd International Conference and Exhibition on Electricity Distribution, CIRED 2013, June 10, (2013)

Shipp D. D., Dionise T. J., Lorch V., Macfarlane W. G., Vacuum Circuit Breaker Transients During Switching of an LMF Transformer, IEEE Transactions on Industry Applications, Vol. 48, No. 1, January/February, (2012)

Stout, J. y Bogh, D., Refiner Autotransformer Motor Start, Pulp and Paper Canada, ISSN: 03164004; Publisher: Southam Inc, v 99, n 6, p 54-58, Jun, (1998)

Telander S.H., Wilhelm M.R. y Stump K.B., Surge Limiters for Vacuum Circuit Breaker Switchgear, IEEE Transactions on Power Delivery, Vol. PWRD-2, No.1, January, (1987)

Vollety C. y De Metz Nobat B., Protecting High Voltage Motors Against Switching Over Voltages, Electrical and Instrumentation Applications in the Petroleum \& Chemical Industry, 4th European Conference on PCIC, PP.1-7, June 1315, (2007)

Wong S.M., Snider L.A., Lo E.W.C., Overvoltages and Reignition Behavior of Vacuum Circuit Breaker, Proc. Of the International Conference on Power System Transients, IPST, New Orleans, Louisiana USA (2003) 